\title{
Comparison of Activation Times Estimation for Potential-Based ECG Imaging
}

\author{
Matthias Schaufelberger ${ }^{1}$, Steffen Schuler ${ }^{1}$, Laura Bear ${ }^{2}$, Matthijs Cluitmans ${ }^{3}$, Jaume Coll-Font ${ }^{4}$, \\ Önder Nazim Onak ${ }^{5}$, Olaf Dössel ${ }^{1}$, Dana Brooks ${ }^{4}$ \\ ${ }^{1}$ Institute of Biomedical Engineering, Karlsruhe Institute of Technology, Karlsruhe, Germany \\ ${ }^{2}$ IHU-LIRYC Electrophysiology and Heart Modeling Institute, Pessac-Bordeaux, France \\ ${ }^{3}$ Maastricht School for Cardiovascular Diseases, Maastricht UMC, Maastricht, Netherlands \\ ${ }^{4}$ Department of Electrical \& Computer Engineering, Northeastern University, Boston, USA \\ ${ }^{5}$ Middle East Technical University, Ankara, Turkey
}

\begin{abstract}
Activation times (AT) represent the sequence of cardiac depolarization and are one of the most important parameters of cardiac electrical activity. However, estimation of $A T s$ is challenging due to multiple sources of noise. If ATs are estimated from signals reconstructed using electrocardiographic imaging (ECGI), additional problems can arise due to over-smoothing or ambiguities in the inverse problem. Resulting AT maps can show falsely homogeneous regions or artificial lines of block. As ATs are not only important clinically, but are also used for evaluation of ECGI, it is important to understand where these errors come from.

We present results from a community effort to compare AT estimation methods on a common dataset of simulated ventricular pacings. ECGI reconstructions were performed in terms of transmembrane voltages as well as epi-endo and pericardial potentials, all using 2nd-order Tikhonov and 6 regularization parameters. ATs were then estimated by the participants and compared to the truth.

While the pacing site had the largest effect on AT correlation coefficients $(C C)$, there were also differences between methods and source models that were poorly reflected in CCs. Results indicate that artificial lines of block are most severe for purely temporal methods. Compared to the other source models, ATs estimated from transmembrane voltages are more precise and less prone to artifacts.
\end{abstract}

\section{Introduction}

Cardiac arrhythmias are characterized by abnormally propagating depolarization waves on the heart. Often, a diagnosis can be made by identifying the propagation sequence of the wavefront in terms of activation times (AT). Body surface potentials (BSP) arise due to the electrical activity of the heart. Reconstructing a representation of this activity non-invasively from BSPs is known as electrocardiographic imaging (ECGI). Seeking to reconstruct the cardiac potentials or voltages from BSPs is the most common approach to ECGI. However, this inverse problem is severely ill-posed and ATs estimated from reconstructions can show unphysiological patterns, artificial lines of block (ALB) and incorrectly homogeneous regions [1-3].

There are many parameters which have an influence on the final AT maps other than the AT estimation method itself. First of all, a good solution to the inverse problem is crucial. To tackle the ill-posedness, regularization is necessary. In this work, second-order Tikhonov regularization was applied and the effect of over- and undersmoothing on resulting ATs was studied by varying the regularization parameter. To assess the influence of different source models, reconstructions were performed for three of the most commonly used source models: (1) transmembrane voltages (TMV) on the epi- and endocardial surface, (2) extracellular potentials (EP) on the epi- and endocardial surface (epi-endo potentials, EEP) and (3) EPs on the pericardial surface (pericardial potentials, PP). Each model has its advantages and disadvantages. In theory, TMVs most directly represent the local activity, as their spatial gradient is the source of electrical potentials on a cellular level. However, this means that each timestep of reconstructed TMVs may have an arbitrary, spatially constant offset. EPs, on the other hand, contain "far" field components and therefore also depend on the activity of surrounding tissue. However, EPs lead to a slighty less ill-conditioned problem than TMVs. Finally, PPs cannot display endocardial activity, but further reduce the complexity of the inverse problem.

As different methods and source models are widely used, a comparison on a common dataset is an important step to gain further insights into the field of non-invasive AT mapping. This study aims to give an overview of established AT estimation methods and their dependency on reconstruction parameters. 


\section{Methods}

\subsection{Simulations and Ground Truth}

Three ventricular pacings at the LV and RV lateral wall (lvL, rvL) and the RV septum (rvS) were simulated using the monodomain model and the ionic model of ten Tusscher et al. (2006). Endo- and epicardial fiber angles were set to $\pm 60^{\circ}$ and cell model variants for endo, mid and epi layers were used. Intracellular conductivities were adjusted to obtain conduction velocities (CV) of 0.6 and $0.3 \mathrm{~m} / \mathrm{s}$ in fiber and transverse directions, respectively. To obtain BSPs and EPs on the epi-, endo- and pericardial surface, the Poisson equation was solved inside a homogeneous and isotropic torso model using the finite element method (mean edge length inside myocardium: $0.53 \mathrm{~mm}$ ).

As previously mentioned, three surface source models were considered for reconstructions: TMVs, EEPs, and PPs. Additionally, two different resolutions of ventricular triangle meshes were used for each model (Res0: mean edge length of $12.42 \mathrm{~mm}, 578$ nodes; Res1: $6.21 \mathrm{~mm}$, 2306 nodes). The pericardial surface was created by keeping the epicardial nodes of the epi-endo surface and adding caps with a similar edge length. Since high frequency components cannot be represented on a coarse mesh, Laplacian blur downsampling [4] was applied to obtain lowresolution ground truth (GT) data for all source models.

\subsection{ECGI Reconstructions}

For reconstructions, BSPs at 200 electrodes were corrupted with 10 different realizations of white Gaussian noise (mean signal-to-noise ratio (SNR) with respect to the depolarization interval: $20 \mathrm{~dB}$ ). The boundary element method was used to compute forward matrices for a torso model equivalent to the finite element model and secondorder Tikhonov regularization was applied:

$$
\widehat{\mathbf{X}}=\arg \min _{\mathbf{X}}\left\{\|\mathbf{A X}-\mathbf{B}\|_{F}^{2}+\lambda\|\mathbf{L X}\|_{F}^{2}\right\}
$$

A is the forward matrix mapping sources to BSPs, $\widehat{\mathbf{X}}$ the estimated source signals in space and time, $\mathbf{B}$ the measured BSPs, $\lambda$ the regularization (smoothing) parameter, and $\mathbf{L}$ an approximation of the Laplacian. The transmural extension of the Laplacian from [5] with Gaussian weighting of volumetric geodesic distances $(\sigma=7 \mathrm{~mm})$ was used for TMVs and EEPs. For PPs, surface distances were used instead. Undersmoothing leads to arbitrary waveforms, whereas oversmoothing destroys high gradients which might be important for AT estimation. Note that $\lambda$ has to be determined for each source model individually, as magnitude, spatial properties and number of sources change between models. To get a meaningful comparison, we therefore used the GT to determine an optimal parameter $\lambda_{\text {opt }}$. As for all sources, the spatial gradi- ent is an important feature for AT estimation and only the gradient of TMVs induces BSPs, we defined:

$$
\lambda_{\mathrm{opt}}=\arg \max _{\lambda}\left\{\underset{\forall i, t}{\operatorname{corr}}\left(\left\|\nabla \widehat{x}_{i, t}(\lambda)\right\|_{2},\left\|\nabla x_{i, t}^{\text {truth }}\right\|_{2}\right)\right\}
$$

$\widehat{x}_{i, t}$ and $x_{i, t}^{\text {truth }}$ are reconstructed and GT sources at node $i$ and timestep $t$, respectively. $\nabla$ is the surface gradient operator and corr denotes the Pearson correlation, which is computed over space and time. As including timesteps with little activity would overestimate $\lambda_{\text {opt }}$, only the depolarization interval as defined by true ATs is used here.

To study the effect of mild and severe over- and undersmoothing, four more values of $\lambda$ were obtained by varying $\log _{10}\left(\lambda_{\text {opt }}\right)$ by \pm 1.5 and \pm 3 , respectively. To assess the performance in realistic scenarios with no GT available, a sixth $\lambda$ was determined using the L-curve method. The depolarization interval used for the L-curve was defined as the time for which the sum of BSP magnitudes across all electrodes is larger than $15 \%$ of its maximum. For most cases, this yielded a $\lambda_{\mathrm{L} \text {-curve }}$ close to $\lambda_{\text {opt }}$.

For TMVs, the baseline correction in [6] was applied after reconstructions to correct for ambiguous spatial offsets.

A total of 1080 reconstructions were performed ( 3 pacings $\times 3$ source models $\times 2$ resol. $\times 10$ noises $\times 6 \lambda$ s).

\subsection{Activation Times Estimation Methods}

\subsubsection{Deflection Based Methods}

Essentialy, deflection based (DB) methods estimate ATs for each point individually by determining the time of a prominent deflection in the local source signal or a signal derived from local sources. These times are also called intrinsic deflection times. ATs can be defined by the maximum positive slope in TMV time courses or the corresponding maximum negative slope in EP time courses (DB temporal, DB-T). While this is a purely temporal marker for each point, attempts have been made to make use of the fact that the depolarization is characterized by a simultaneous gradient of sources in space and time [7]. Using a spatiotemporal gradient signal can increase the SNR for AT estimation and resolve ambiguities in the purely temporal derivative (DB spatiotemporal, DB-ST).

\subsubsection{Cross-Correlation Based Methods}

Using delays between adjacent nodes, cross-correlation based (CB) methods link time and space to estimate the relative AT map at once with an undetermined offset [8]. The delay of each node pair is determined using temporal cross correlation of the temporal derivative (CB temporal, $\mathrm{CB}-\mathrm{T}$ ) or the spatiotemporal derivative (CB spatiotemporal, $\mathrm{CB}$ $\mathrm{ST})$. While there are also approaches to combine $\mathrm{DB}$ and CB methods using a confidence weighting [2], only results for the purely $\mathrm{CB}$ approach from [8] are shown. 


\section{Results}

Fig. 1 shows the GT for each pacing. AT maps in Fig. 2, 3 and 4 were estimated from reconstructions and scaled as the corresponding GT. Numbers above AT maps represent mean and standard deviation of the Pearson correlation coefficient (CC) over all noise realizations. For PPs, the correlation was computed only for pericardial points where GT ATs are available (excluding the caps). Overand undersmoothing refers to the mild variation of $\lambda_{\text {opt }}$.

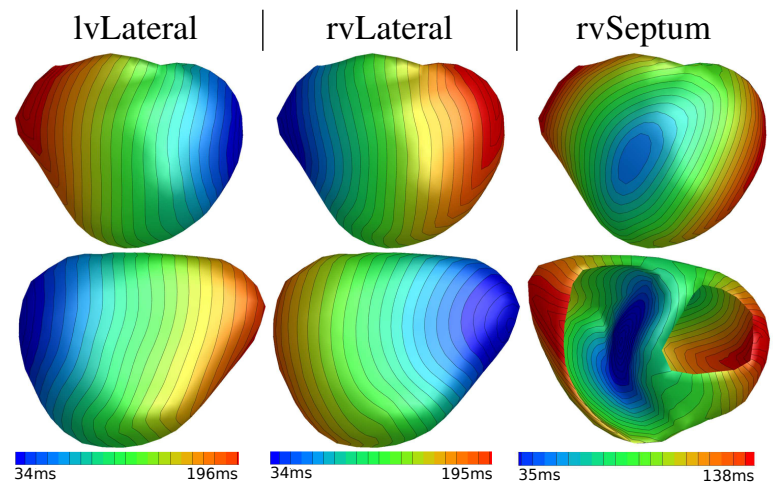

Figure 1: Ground truth ATs for all three pacings.

Fig. 2 depicts results for DB methods. DB-T led to large homogeneous regions separated by high gradients. Especially for the case of oversmoothing, these might be misinterpreted as ALBs. The DB-ST approach improved CCs and better recovered the correct pattern. Although Fig. 2 only depicts EEPs, both TMVs and PPs yielded a similarly "patchy" representation for DB-T methods. As the resolution showed only minor effects on global AT patterns, only Res 1 is depicted in Fig. 3 and 4.

Fig. 3 shows results for all three source models and different levels of smoothing. DB-ST and CB-ST methods were applied for each configuration. TMVs consistently yielded highest CCs and showed fewer ALBs. For the lateral pacings, TMVs appeared to be robust regarding underand oversmoothing. While undersmoothing was best for EEPs, PPs benefitted from oversmoothing. Due to the relative nature of inter-node delays, CB-ST results consistently showed higher absolute errors than DB-ST results regardless of the source model.

Fig. 4 depicts the best methods for the difficult rvS pacing: For TMVs this was a DB-ST method, for EEPs a CBST method, and for PPs a DB-ST method. TMVs showed the highest CCs and indicated pacing origins close to the septal wall. For the optimal smoothing, two pacing origins appeared. For under- and oversmoothing, there was only one origin wrongly located at the RV anterior wall. EEPs created unphysiological patterns regardless of the reconstruction. Although PPs cannot represent endocardial activity, AT maps resembled the results obtained from TMVs on the epicardium, but with a similarly low CC as EEPs.

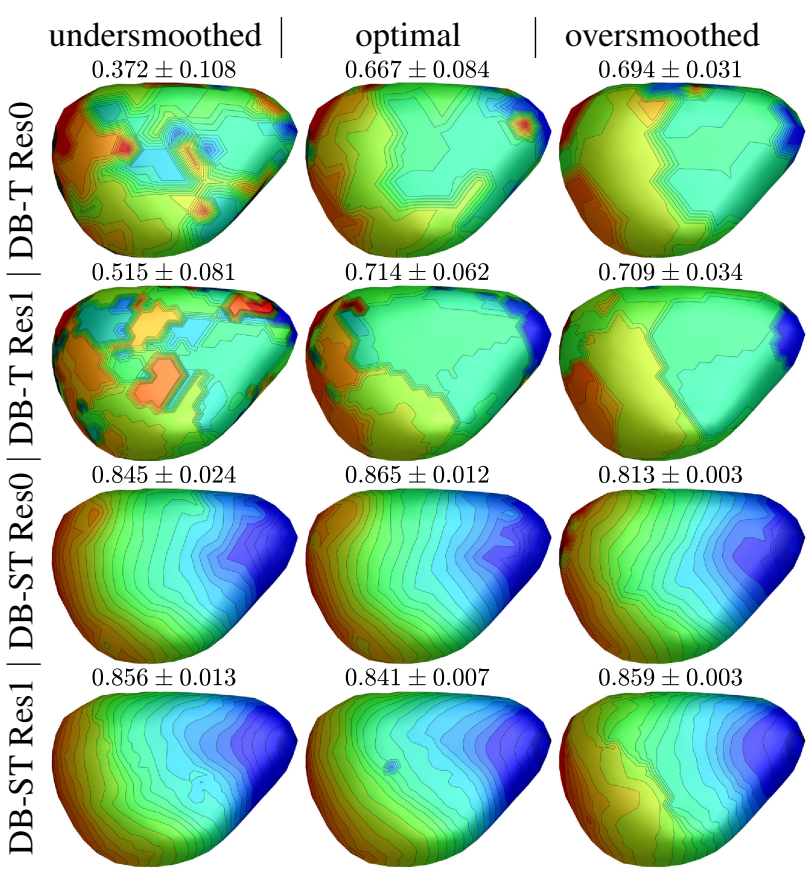

Figure 2: EEP comparison of temporal and spatiotemporal AT estimation for the rvL pacing and both resolutions.

\section{Discussion and Conclusion}

Overall, TMVs yielded the best CCs for all pacings and showed fewest ALBs. Spatiotemporal methods outperformed purely temporal ones regarding $\mathrm{CCs}$ and occurences of ALBs. DB and CB methods performed similarly. Even for epicardial points, EEPs performed slightly better and more robust than PPs. Results indicate that ALBs resulting for EPs in particular might be a consequence of "averaging" signals with spatially changing morphology during regularization. The morphology of TMVs changes less across space than for EPs. This might explain why they are less prone to artifacts in ATs. More research is planned regarding the true cause of ALBs.

This work has several limitations. To compensate for not including mismatches in the forward model, we added more white Gaussian noise than typically present in BSPs. Especially DB-T methods might suffer from this low SNR. Different errors might occur in a clinical setting with imperfect geometries and less uncorrelated noise. Neither scars, nor areas with lower CV were included in the simulation. Further analysis is necessary to determine the performance of the different source models and estimation methods regarding the detection of real scars and not only the absence of ALBs.

Since only second-order Tikhonov regularization was evaluated, results might differ for other regularization techniques. For TMVs, the influence of the baseline correction should be investigated, e.g. using a purely spatial method. 


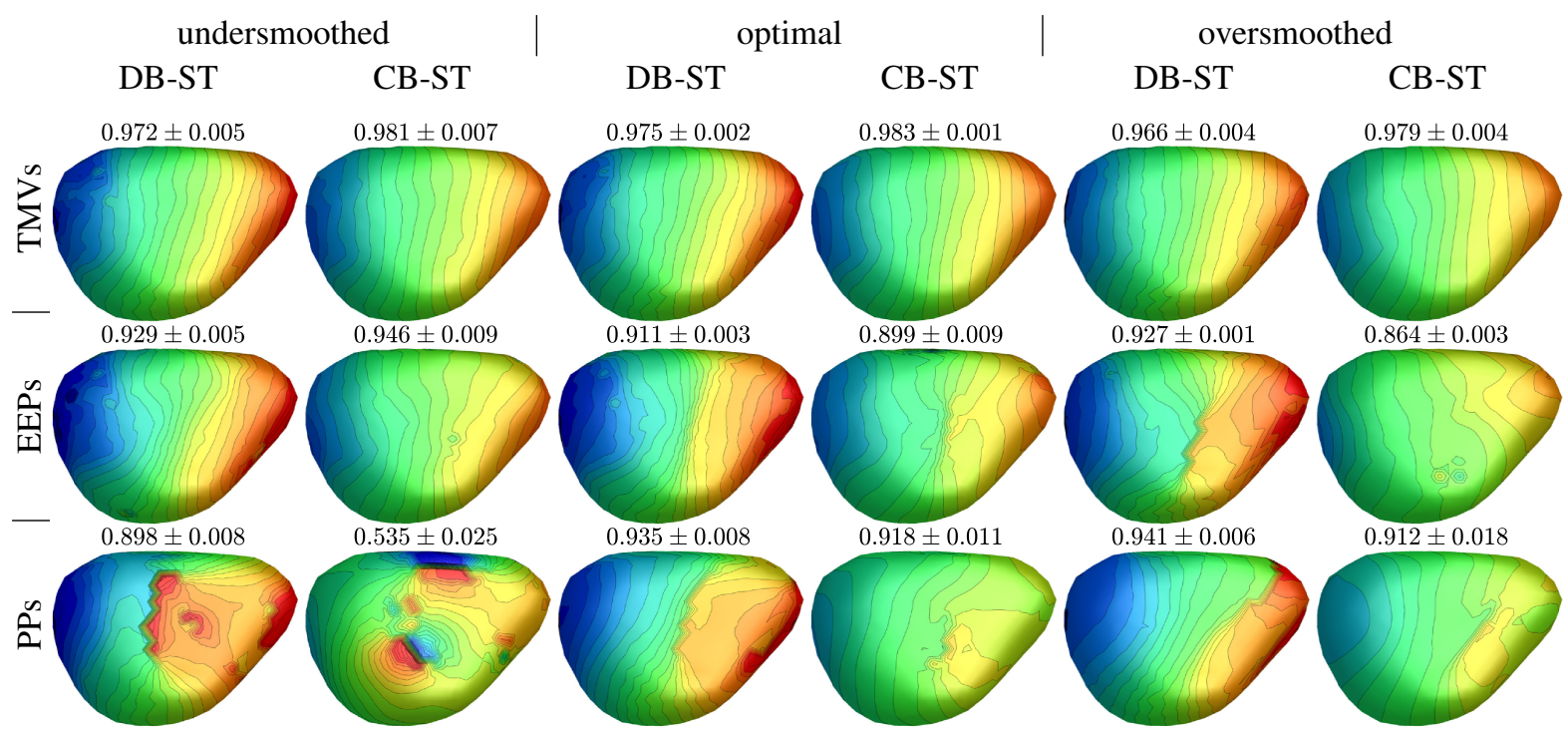

Figure 3: Comparison of source models and deflection and cross-correlation based methods for the lvL pacing. undersmoothed
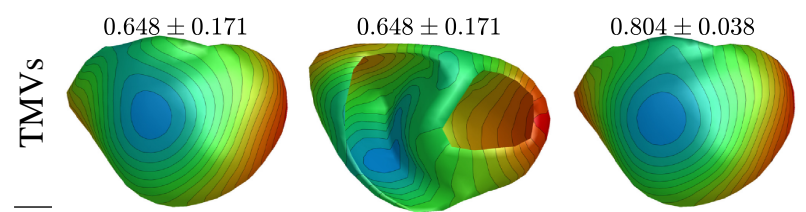

optimal

oversmoothed
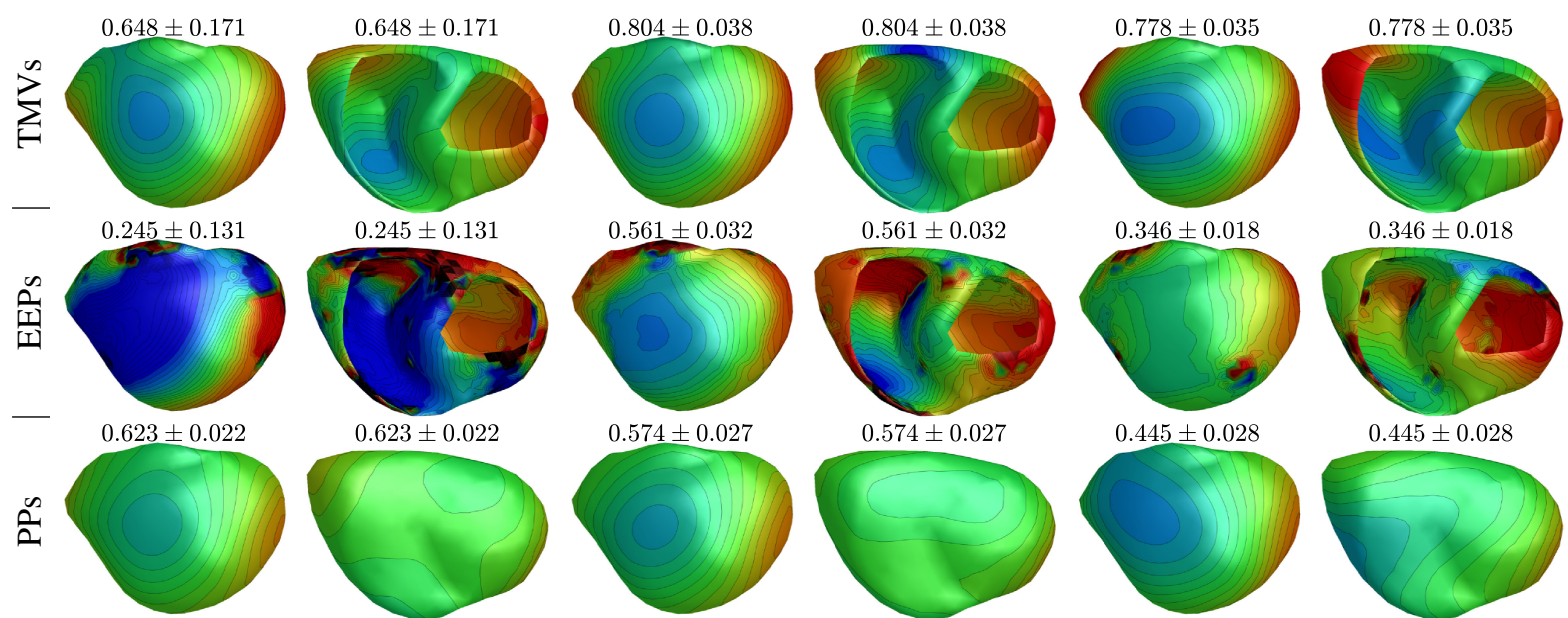

$0.561 \pm 0.032$
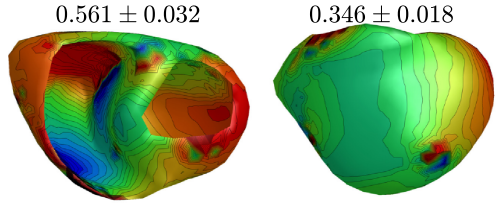

$0.346 \pm 0.018$
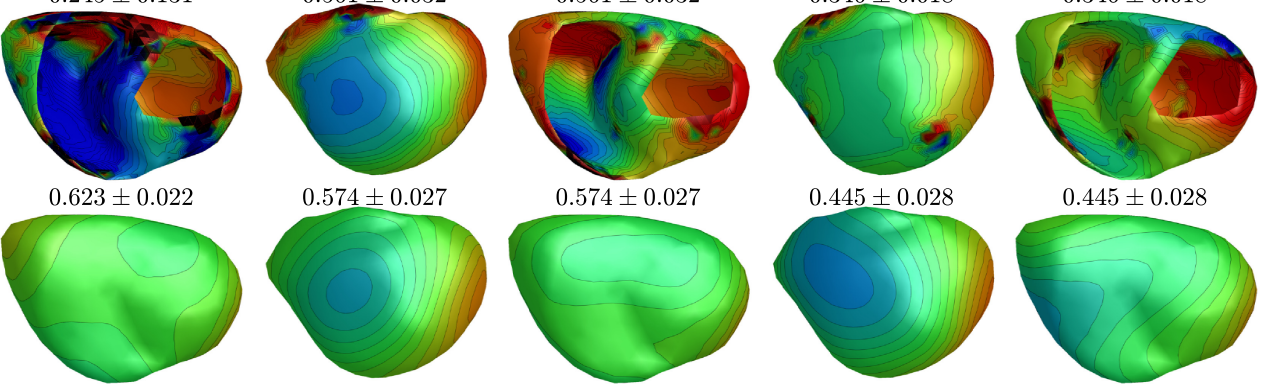

Figure 4: Comparison of best AT estimation methods for each source model for the rvS pacing (front and top view).

\section{References}

[1] Duchateau J, Sacher F, Pambrun T, Derval N, et al. Performance and limitations of noninvasive cardiac activation mapping. Heart rhythm 2019;16(3):435-442.

[2] Duchateau J, Potse M, Dubois R. Spatially coherent activation maps for electrocardiographic imaging. IEEE Transactions on Biomedical Engineering 1 2017;64(5):1149-1156.

[3] Bear LR, Bouhamama O, Cluitmans M, Duchateau J, et al. Advantages and pitfalls of noninvasive electrocardiographic imaging. J Electrocardiol 2019;.

[4] Schuler S, Tate JD, Oostendorp TF, MacLeod RS, Dössel O. Spatial downsampling of surface sources in the forward problem of electrocardiography. In FIMH, volume 11504 of LNCS. Springer, 2019; 29-36.

[5] Erem B, Coll-Font J, Orellana RM, Stovicek P, Brooks DH. Using transmural regularization and dynamic modeling for noninvasive cardiac potential imaging of endocardial pacing. IEEE Trans Med Imaging 2014;33(3):726-738.

[6] Schuler S, Potyagaylo D, Dössel O. Delay-based regularization for ecg imaging of transmembrane voltages. In Comput Cardiol, volume 46. 2019; .

[7] Erem B, Brooks DH, van Dam PM, Stinstra JG, MacLeod RS. Spatiotemporal estimation of activation times of fractionated ecgs on complex heart surfaces. In Eng Med Biol Soc. IEEE, 2011; 5884-5887.

[8] Dubois R, Labarthe S, Coudière Y, Hocini M, Haïssaguerre M. Global and directional activation maps for cardiac mapping in electrophysiology. In Comput Cardiol, number 39. 2012; 349-352.

Address for correspondence:

Matthias Schaufelberger, publications@kit.edu Fritz-Haber-Weg 1, 76131 Karlsruhe, Germany 